Dr Lucas comments:

I welcome the comments of Drs McWilliam and Curry on the problems of diagnosing microsporidiosis in small intestinal biopsy specimens. Of course, the final arbiter is properly prepared electron microscopy. The "probable spores" in our cases were "probable" on electron microscopic examination of very limited quantities of material originally taken to paraffin wax, then reprocessed. The important point is that the spores seen by light microscopy of smears stained with haematoxylin and eosin in the case of microsporidosis definitely confirmed by electron microscopy were identical with those seen in stained sections of our other four cases (our figs 2 and 3 showed different patients). Thus I feel confident in making the diagnosis when these characteristic intracellular bodies are seen

If they are not seen by light microscopical examination the diagnosis of microsporidiosis cannot be excluded. Sampling is one reason. Also, in my experience only the spore and spore-forming stages of the life cycle of intestinal microsporidia can be seen on standard haematoxylin and eosin staining; the meront stages (which may be more numerous than spores) are essentially invisible, only being identified by electron microscopy.

Finally, a large series of small intestina biopsy specimens from American homosexual HIV positive patients was reported at a recent AIDS conference. Of 71 biopsy specimens from 67 patients, 22 showed microsporidia by transmission electron microscopy. Seventeen of these 22 positive cases were also identified on semithin sections. Tantalisingly, the authors say, "in retrospect, parasites were also visible by light microscopy in many of the standard haematoxylin and eosin stained sections'. We eagerly await the definitive publication.

I think that pathologists can spot these protozoa in many instances in ordinary sections, but as Drs McWilliam and Curry say, we certainly need more experience of this parasite, with confirmation by electron microscopy.

\section{Peliosis thymomis}

Peliosis thymomis is probably a misnomer. The latin translation of peliosis of a thymoma is peliosis thymomatis.

E CALLEWAERT Algemeen Ziekenhuis St Lucas-St Joze 8320 Brugge 4, Belgium

1 Peliosis thymomis: association with tuberculosis. J Clin Pathol 1989;42:331.

Rapid urease tests for Campylobacter pylori

We read the reply of Vaira, Holton, and Salmon to the points raised by us in our paper.' The authors state that, "the results of $2{ }^{\prime \prime}{ }^{\circ}$ urea test, $6{ }^{\prime}$ " urea test (CP test), and CLO test were done at five, 10 , and 20 minutes. The results at one, three, and 24 hours are also given in our letter'. Vaira et al have reported the results with these three tests (table 2) at five, 10 , and 20 minutes, one hour, two hours, and 24 hours in an in vitro urease test of $C$ pylori, Proteus, and Klebsiella strains, and not an in vivo study using mucosal biopsy specimens. ${ }^{2}$ As we pointed out, in the in vivo study Vaira et al read the results of the CLO test at 20 minutes, 90 minutes, and 24 hours, results of the $2^{\circ}$ 。 RUT at three hours, four hours, and six hours, results of the CP test at 15 minutes, 20 minutes, and two hours. In their subsequent letter the authors reported the sensitivity and specificity of various tests, but comparison of sensitivity and specificity of various tests at different time intervals was not described either in the tables or in the text. ${ }^{2}$

The original letter of the authors ${ }^{3}$ concerns the four hour urease test which uses $2^{\circ}$ urea broth and incubation at $37^{\circ} \mathrm{C}$. The text of this letter gave the sensitivity and specificity of the test at four hours, but as the test was read at a fixed interval of four hours, the authors' point that they gave the sensitivity and specificity of tests at different times in the text of their original letter does not hold true.

We had raised these basic points to arrive at an understanding of whether the results with different types of rapid urease tests with different media should be read at different time intervals or at the same fixed interval. The point remains unanswered.

$$
\begin{array}{r}
\text { DK BHASIN } \\
\text { A AYYAGIRI } \\
\text { V SINGH } \\
\text { SK MEHTA } \\
\text { Departments of Gastroenterology and } \\
\text { Microbiology, Postgraduate Institute of } \\
\text { Medical Education and Research, } \\
\text { Chandigarh, India }
\end{array}
$$

1 Bhasin D, Yachha S, Ayyagiri A, et al. How specific is rapid urease test for diagnosing specific is rapid urease test for diagr
C pylori? J Clin Pathol 1989;42:671.

2 Vaira D, Holton J, Cairns S, et al. Urease tests for Campylobacter pylori: care in interpretation. J Clin Pathol 1988;41:812-3.

3 Vaira D, Holton J, Cairns S, Falzon M, Salmon $P$. Four hour rapid urease test (RUT) for detecting Campylobacter pylori: is it reliable enough to start treatment? J Clin Pathol $1988 ; 41: 355-6$

\section{A five minute stain for Campylobacter} in tissue

Gray et al described a 30 minute stain for Campylobacter using $2^{\circ}$ ". Giemsa without differentiation.' We have also found this method successful. By chance we also used stronger stain with shorter times of staining. The results were better because there was less background staining.

Our technique is to stain with $20^{\circ}$ "Giemsa for five minutes, then blot dry on filter paper, and very quickly dehydrate in one jar of absolute alcohol and transfer to Xylol before any loss of stain has occurred into the alcohol. After five minutes the staining of the Campylobacter is as heavy as that after longer staining. An advantage of the shorter staining time is that the background mucin stains less intensely, as do the gastric glands. As a result Campylobacter can be seen deep in the mucin of the gland crypts as well as in the mucin on the surface. The $20^{\circ}{ }_{0}$ Giemsa has a bench life of a month if kept at room temperature.

CR ROBINSON Institute of Pathology Soroha Hospital, Beer Shera, Israel

1 Gray SF, Wyatt JI, Rathbone BJ. Simplified technique for identifying Campylobacter pyloridis. J Clin Pathol 1986;39:1279-80.

\section{BOOK REVIEWS}

A Colour Atlas of AIDS and HIV Disease. 2nd ed. CF Farthing, $S$ Brown, RCD Staughton. (Pp 115; £16.) Wolfe Medical Publications. 1988. ISBN 0-7234-0995-1.

The first edition of this book was produced in 1986 , and in the intervening two years until the availability of this edition experience of HIV disease has been broadened. This is well reflected in this edition which includes a more wide ranging account of the clinical manifestations of HIV disease. The appendices have also been expanded to cover important topics including the full Centers for Disease Contro definition of AIDS and advice for people who are HIV antibody positive.

As with the first edition, the pictures are all of high quality, which, when coupled with the concise text, make this book highly recommended reading for all health care personnel who are interested in, or more especially involved with, the care of people with HIV disease and AIDS

P GRINT

Disorders of the Spleen. Major Problems in Pathology. Vol. 20. Barbara C Wolf and Richard S Neiman. (Pp 211; £37.50.) WB Saunders. 1989. ISBN 0-7216-2503-7.

Most pathologists become decidedly uneasy when called on to interpret the histology of the spleen, not least because definitive accounts of splenic pathology are few and far between. This book goes a long way towards remedying this deficit. The information is presented in a precise and well ordered fashion and in most areas is admirably up to date and comprehensive: it is also supplemented by many helpful diagrams, tables, and references. Indeed it is difficult to find omissions, although more data regarding the hairy cell leukaemia variants-possibly the most common primary splenic neoplasmswould have been useful. It must also be said that not all of the photomicrographs (mainly in black and white) are wholly successful and some would have benefited from higher magnification.

All the same these are minor complaints and most pathologists, haematological or otherwise, will welcome this most creditable account of splenic pathology and might at last begin to make some sense out of an organ which hitherto has jealously guarded its mysteries.

F D LEE

Recent Advances in Clinical Pharmacology and Toxicology. No, 4. Ed P Turner, GN Volans. (Pp 276; \$45.) Churchill Livingstone. 1989. ISBN 0-44303297-5.

Five years have elapsed since publication of the last Recent Advances in Clinical Pharmacology, and in producing the latest volume the editors have taken the initiative to broaden its scope to include recent advances in the field of "clinical toxicology". Thus a very wide and often puzzling array of subject areas are covered in the present volume. For example, the first of the 13 essays is an 
account of the application of positron emission tomography to the identification of opiate receptors in the human brain. This chapter, however, fails to point out the much greater potential of the technique to clinical pharmacology, provided that the investigator has access to a cyclotron to produce the necessary short lived isotopes. I found the next chapter, which deals essentially with the pharmacodynamics of antihypertensive drugs, equally complex - not from a technological viewpoint but rather from the authors' stoical treatment of the subject. Similarly, chapter 7 is an unmoving account of the "clinical pharmacology of skin" which is a shame because "skin is one of the larger human organs and acts as a barrier between internal and external environments." This chapter could have interfaced well with chapter 11 on "Pesticide use-risk or safety" because the dividing line is often the pesticide's dermal absorption. Thus in short, some general guidance/overview of the contents in the form of a foreword by the editors would have been very much appreciated by this reader. I could relate much more easily to the more dynamic and personalised chapters dealing with atrial natriurecic factor, prostaglandins, and the gastrointestinal tract, the use of nitrates in treatment of angina etc, as well as that on drugs and male reproductive function. Finally, the chapters dealing with genetic polymorphisms underlying iatrogenic disease, dealing with the role of oncogenes in human carcinogenesis, describe the frontiers of the molecular approach to modern medicine. Sandwiched in between these intellectually stimulating layers are reasonable accounts of adverse reactions to drugs in the elderly, the use of antidotes in selected cases of human poisoning, and an overview of aluminium toxicity.

In general, the volume and its editors are to be commended for undertaking a Herculean task resulting from the incorporation of a multidisciplinary subject such as toxicology into the series title.

Although this may broaden the appeal of the series, its contents inevitably embrace the adage that "one man's meat is another's poison."

A PAINE

Cytopathology of Soft Tissue and Bone Tumors. Monographs in Clinical Cytology Vol 12. SI Hajdu, EO Hajdu. (Pp 348; 495 figs $£ 100$.) Karger. 1989. ISBN 3-80554814-1.

This book is a well written addition to the Monographs in Clinical Cytology series and is a thoroughly researched guide to the myriad soft tissue and bone lesions that abound. The first chapter, which gives a detailed account of histogenesis and classification, and the appendices listing differential diagnoses, immunochemical reactions, and ultrastructural features of soft tissue "tumours," make this an ideal reference book for pathologists.

An interesting aspect is the combination of $x$ ray appearances, clinical features, and detailed histological findings in most of the conditions discussed. Only a small number of the entities described, however, have detailed cytological descriptions and photomicrographs, indirectly emphasising the fact that a definitive diagnosis of soft tissue neoplasms is nearly impossible in fine needle aspiration cytology.
Unfortunately this book does not fulfil its goal-to be a useful guide on the cytopathology of soft tissues and bones-as it contains very little on typical cytological features seen in fine needle aspirates.

GRACE MCKEE

Genetic Analysis of Tumour Suppression. Ciba Foundation Symposium 142. (Pp 258; £32.50.) John Wiley. 1989. ISBN 0471922994.

Recorded here are the proceedings of some thoughtful and obviously lively debates following formal presentations by scientists who have made major contributions to work related to tumour suppression. Each paper reviews past results and often previews current results from different laboratories.

The phenomenon of tumour suppression was first identified 20 years ago in somatic cell hybrids between normal and malignant cells: the hybrids proved not to be malignant. Henry Harris and George Klein made those initial observations and both men contribute to this symposium. A large number of tumour suppressor genes have been identified in Drosophila. One of the best described is the l( 2)gl gene: Bernard Mechler presented his laboratory's recent results suggesting that the critical period for the establishment of tumourigenesis in $l(2) g l$ mutants occurs very early in development. Much excitement has attended the first cloning in Robert Weinberg's laboratory of a human tumour suppressor gene, $R b$, associated with retinoblastoma. Results he presented suggest that the $R b$ mutation leads to non-expression of a receptor for a negative regulatory growth factor on the surface of fetal retinal cells.

There are more than a dozen examples of mapped loci where loss of genetic information predisposes to different cancers. With rapid advances in both human genome mapping techniques and reverse genetics, it is likely that many more genes will be identified and new insights gained into tumourigenesis.

This Ciba Symposium makes a spirited and welcome contribution to what George Klein has called this emerging era of tumour suppressor genes. The book will be useful to those entering this field who seek a topical overview.

MAR YUILLE

Cell Kinetics of the Inflammatory Reaction. Ed CL Berry, E Grundemann. Current Topics in Pathology. Vol 79. (Pp 320; DM 190.) Springer. 1989. ISBN 3-540-19459-2.

The volume consists of a series of short reviews on the kinetics of the major cell types involved in the inflammatory reactionneutrophil granulocytes, $B$ and $T$ lymphocytes, dendritic leucocytes, monocyte macrophages and mast cells. Each chapter deals with the origin, differentiation, migration, functional role and mode of elimination of a particular cell type and its principal interactions with other cell types and physiological control mechanisms. The editor has been successful in persuading his eminent contributors to give comprehensive papers without undue overlap or gross omissions. The volume gives a balanced overview that will make it particularly valuable to newcomers to the field of cell kinetics of inflammation, but the individual chapters will also be useful to established workers because all are informed critical reviews with informative contemporary references.

The chapter on molecular events in liver regeneration is a scholarly review, but it is misplaced as its presumed audience is unlikely to read a book on inflammation.

J SWANSON-BECK

Medical Mycology. A Practical Approach. The Practical Approach Series. Ed EGV Evans, MD Richardson. (Pp 300; paperback $£ 25$; hardback $£ 37 \cdot 50$.) Oxford University Press. 1989. ISBN 0199630119 (paperback) and 0199630100 (hardback).

In this book the techniques for the isolation culture, and identification of yeasts and filamentous and dimorphoic fungi capable of causing human infection are comprehensively described. Chapters on the identification of culture contaminants, fungal serology, assay of antifungal drugs and histopathology of mycoses make this book a complete manual for laboratory investigation of fungal diseases.

Subdivision of each of the 12 chapters into numbered sections makes every stage of a procedure easy to follow and facilitates quick cross reference. All techniques are described in detail, including the composition of media, reagents, and stains. The text is well illustrated with diagrams and black and white photographs. Colour plates in chapters describing colony morphology and histopathology would have been very welcome.

All 15 authors (predominantly from the United Kingdom) are highly regarded mycologists whose critical choice of methods has clearly arisen from their own personal experience. The editors are to be congratulated in presenting this expertise in a well structured format which makes this book a requisite for all laboratories wishing to undertake the diagnosis of fungal infections.

GR JONES

A Colour Atlas of the Digestive System. RE Pounder, MC Allison, AP Dhillon (Pp 232; $£ 30.00$.) Wolfe Medical Publications Ltd. 1989. ISBN 0723408866 .

This atlas illustrates all aspects of the digestive system, including mouth, oesophagus, stomach, duodenum, pancreas, small and large intestine, appendix, and anus. Skin, stomas, and hernias are also included. The liver and biliary system are not covered (a comparison volume by Sherlock and Summerfield does this), although there are ERCP radiographs of sclerosing cholangitis and haemobilia.

Atlases suffer from the inherent problem of only being able to cover their field in a superficial way and this one is no exception. This atlas, however, scores by the wide range of its coverage. Of particular interest to pathologists will be the clinical photographs of such conditions as acanthosis nigricans, Grey-Turner's sign in acute pancreatitis, dermatitis herpetiformis, pellagra, typhoid, and various Kaposi lesions. There are also particularly good pictures of a variety of worms and anal lesions. There are good examples of computed tomography scans and 